\title{
Differential Gene Expression during Trypanosoma cruzi Metacyclogenesis
}

\author{
Marco Aurelio Krieger, Andrea Rodrigues Ávila, Sueli Fumie Yamada Ogatta, \\ Claire Plazanet-Menut, Samuel Goldenberg ${ }^{+}$
}

\author{
Departamento de Bioquímica e Biologia Molecular, Instituto Oswaldo Cruz, Avenida Brasil 4365, 21045-900 \\ Rio de Janeiro, RJ, Brasil
}

The transformation of epimastigotes into metacyclic trypomastigotes involves changes in the pattern of expressed genes, resulting in important morphological and functional differences between these developmental forms of Trypanosoma cruzi. In order to identify and characterize genes involved in triggering the metacyclogenesis process and in conferring to metacyclic trypomastigotes their stage specific biological properties, we have developed a method allowing the isolation of genes specifically expressed when comparing two close related cell populations (representation of differential expression or RDE). The method is based on the PCR amplification of gene sequences selected by hybridizing and subtracting the populations in such a way that after some cycles of hybridization-amplification genes specific to a given population are highly enriched. The use of this method in the analysis of differential gene expression during T. cruzi metacyclogenesis $(6 \mathrm{hr}$ and $24 \mathrm{hr}$ of differentiation and metacyclic trypomastigotes) resulted in the isolation of several clones from each time point. Northern blot analysis showed that some genes are transiently expressed (6 hr and $24 \mathrm{hr}$ differentiating cells), while others are present in differentiating cells and in metacyclic trypomastigotes. Nucleotide sequencing of six clones characterized so far showed that they do not display any homology to gene sequences available in the GeneBank.

Key words: Trypanosoma cruzi - gene expression - differentiation - metacyclogenesis

The differentiation of epimastigotes into metacyclic trypomastigotes (metacyclogenesis) involves the transformation of a replicative noninfectious form of Trypanosoma cruzi into a nonreplicative infectious stage (De Souza 1983). The functional and morphological changes occurring during this process result from important changes in the gene expression program (Contreras et al. 1985a). As yet few T. cruzi genes have been undoubtedly assigned as displaying a stage specific expression pattern during the metacyclogenesis process. In fact, relatively few $T$. cruzi stage specific genes have been described (Schenkman et al. 1994, Ruef et al. 1994, Teixeira et al. 1994) and most of them encode major antigens.

\footnotetext{
This work received financial support from Pronex, PADCT, CNPq and Fiocruz-Papes. SG is a research fellow from $\mathrm{CNPq}$, ARA is a doctoral of $\mathrm{CNPq}, \mathrm{CP}-\mathrm{M}$ is a post-doctoral of CNPq and SFYO a doctoral Capes fellowship.

${ }^{+}$Corresponding author. Fax: +55-21-590.3495. E-mail: sgoldenb@gene.dbbm.fiocruz.br

Received 9 June 1999
}

Accepted 9 August 1999
The availability of stage specific genes should improve our knowledge of $T$. cruzi differentiation and in particular of the metacyclogenesis process. The study of transiently expressed genes should allow the identification of factors and processes modulating and controlling the differentiation process. On the other hand, the study of metacyclic trypomastigote stage specific genes might be important for understanding the pathology of Chagas disease and might ultimately lead to a better understanding of the mechanisms controlling their expression.

\section{RESULTS AND DISCUSSION}

Important progresses have been described in the techniques aiming to isolate cell type or stage specific genes (Liang \& Pardee 1992, Lisitsyn, et al. 1993). Our recent work resulted in the development of a method suitable for isolating trypanosomatid specific genes (Krieger \& Goldenberg 1998). The method, named RDE for representation of differential expression, consists in the selection of specific genes through PCR amplification of hybrid selected sequences. The method is schematically represented in Fig. 1. Briefly, DNA sequences from a "tester" population, to which a specific set of adaptors is added, are hybridized to an excess of "driver" DNA in order to sequester from the tester all (or at least 
most) sequences common to the driver. Following hybridization, the molecules are amplified by PCR using primers complementary to the adaptors added to the tester DNA. Molecules represented only in tester will be exponentially amplified, those common to tester and driver will be linearly amplified and those exclusive to the driver will not be amplified since they lack the adaptors. After several cycles of hybridization and PCR amplification of selected sequences, the population is highly enriched in DNA sequences specific to the tester population. The primers used for cDNA synthesis are complementary to the 5'-mini-exon (spliced leader) sequence common to all trypanosomatid mRNAs (Boothroyd \& Cross 1988) and to the 3'-poly-A tail. Hence, there are two important points of this method: (i) most (if not all) amplified sequences represent full-length cDNAs and (ii) the fact that this method uses the mini-exon or spliced-leader sequence as a target for PCR-amplification renders this method suitable for any trypano-somatid.

On the other hand, the study of $T$. cruzi metacyclogenesis has been rendered easiest since chemically defined conditions mimicking the process occurring within the triatomine insect have been developed (Contreras et al. 1985b). Epimastigotes from late exponential growth phase are removed from LIT medium and incubated in the chemically defined TAUP or TAU3AAG medium. Following transfer to the chemically defined differentiation medium, epimastigotes adhere to a substrate (glassware) and after $72 \mathrm{hr}$ to $120 \mathrm{hr}$, depending upon the T. cruzi strain, transform into the infective metacyclic trypomastigotes and are released to the culture supernatant (Bonaldo et al. 1988). Hence, we can define several parasite populations according to their time of incubation in the differentiation medium. For the purposes of our studies we have used as tester, cDNAs synthesized from polysomal mRNAs isolated from parasites of $6 \mathrm{hr}, 24 \mathrm{hr}$ of differentiation and metacyclic trypomastigotes, and as driver cDNAs synthesized from epimastigote polysomal mRNAs. With this procedure we attempted to clone genes expressed by differentiating cells and/or metacyclic trypomastigotes and not expressed by epimastigotes.

Several clones have been isolated from each differentiation time, as depicted on Table, and are being presently characterized. Most of the genes sequenced so far did not display any homology to genes whose sequences are available in the GeneBank. The only exception is a gene specifically expressed by differentiating (adhered) epimastigotes that displays a high homology to an unidentified reading frame described in S. cerevisae and in C. elegans. The study of these genes is very interesting because they are stage specific and encode non-structural (major) antigens. Hence, they might encode regulatory proteins involved in cellcycle regulation, proteins involved in gene expression modulation such as transcription factors or special enzymatic activities that might serve as targets for developing new drugs against Chagas disease.

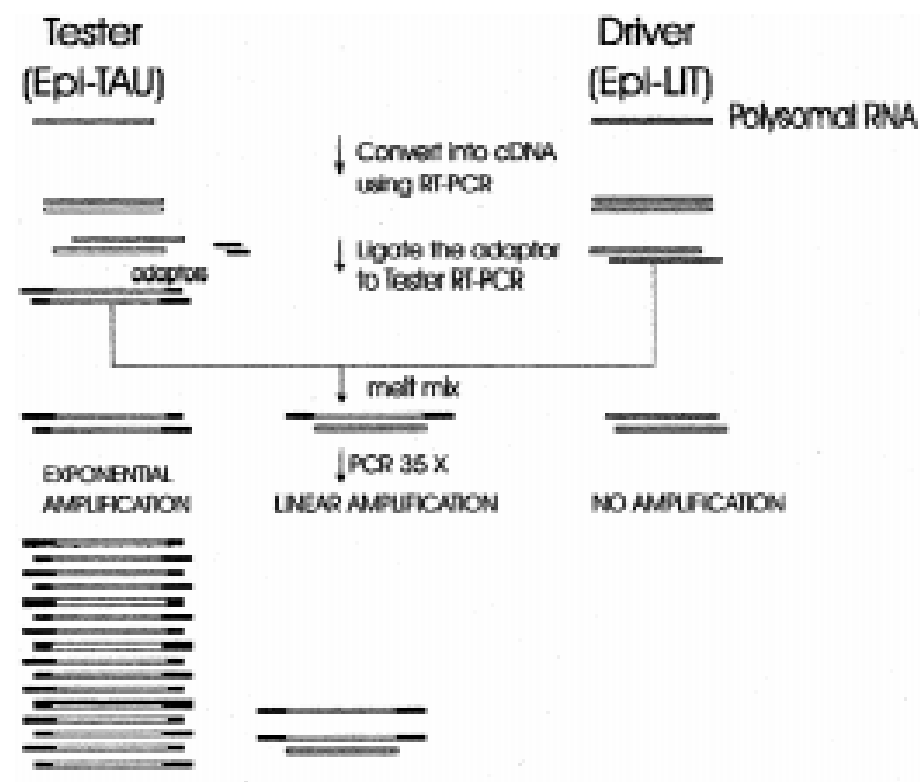

Fig. 1: schematic representation of the RDE (representation of differential expression) method. 
TABLE

Trypanosoma cruzi stage specific clones isolated in the course of the metacyclogenesis process

\begin{tabular}{lcc}
\hline Parasite population & $\begin{array}{c}\text { Number } \\
\text { of clones } \\
\text { sequenced and } \\
\text { characterized }\end{array}$ & $\begin{array}{c}\text { Number } \\
\text { of clones } \\
\text { selected and } \\
\text { under analysis }\end{array}$ \\
\hline LIT medium epimastigotes $^{2} \mathrm{hr}$ differentiating cells & \\
24 & 2 & 59 \\
$24 \mathrm{hr}$ differentiating cells & \\
Metacyclic trypomastigotes & 2 & 26 \\
\hline Total & 2 & 57 \\
\hline
\end{tabular}

$a$ : parasites adhered to the culture flask.

Northern blot analysis of the genes isolated so far showed that some of them are transiently expressed during the metacyclogenesis process while others are expressed by differentiating cells and by metacyclic trypomastigotes, as shown in the results presented in Fig. 2. Clone \#10 starts to be expressed at $24 \mathrm{hr}$ of differentiation and is present in metacyclic trypomastigotes whereas clone \#24 is expressed exclusively by differentiating cells ( 6 $\mathrm{hr}$ and $24 \mathrm{hr}$ parasites).
The availability of a large number of genes whose expression is developmentally regulated should allow important progresses in understanding the mechanisms involved in gene expression regulation in trypanosomatids. Most (if not all) trypanosomatid genes are transcribed as polycistronic pre-mRNAS that are processed for the 5'spliced leader and 3'-poly-A addition (Muhich \& Boothroyd 1988, Lebowitz et al. 1993, Vanhamme \& Pays 1995, Furger et al. 1997). The sequencing of intergenic regions of stage specific genes might reveal motifs involved in gene expression regulation. Furthermore, the study of the 5'- and 3'untranslated regions (UTR) of these genes might contribute to our understanding of their role in gene expression regulation in trypanosomatids (Nozaki \& Cross 1995, Vanhamme \& Pays 1995, Furger et al. 1997).

One of the most promising perspectives about the study of stage specific genes in T. cruzi is their functional characterization. The fact that the developmentally regulated genes we have sequenced so far display no homology to known-genes suggests that these genes might be implicated in controlling the metacyclogenesis process (for transiently expressed genes) or alternatively in con-

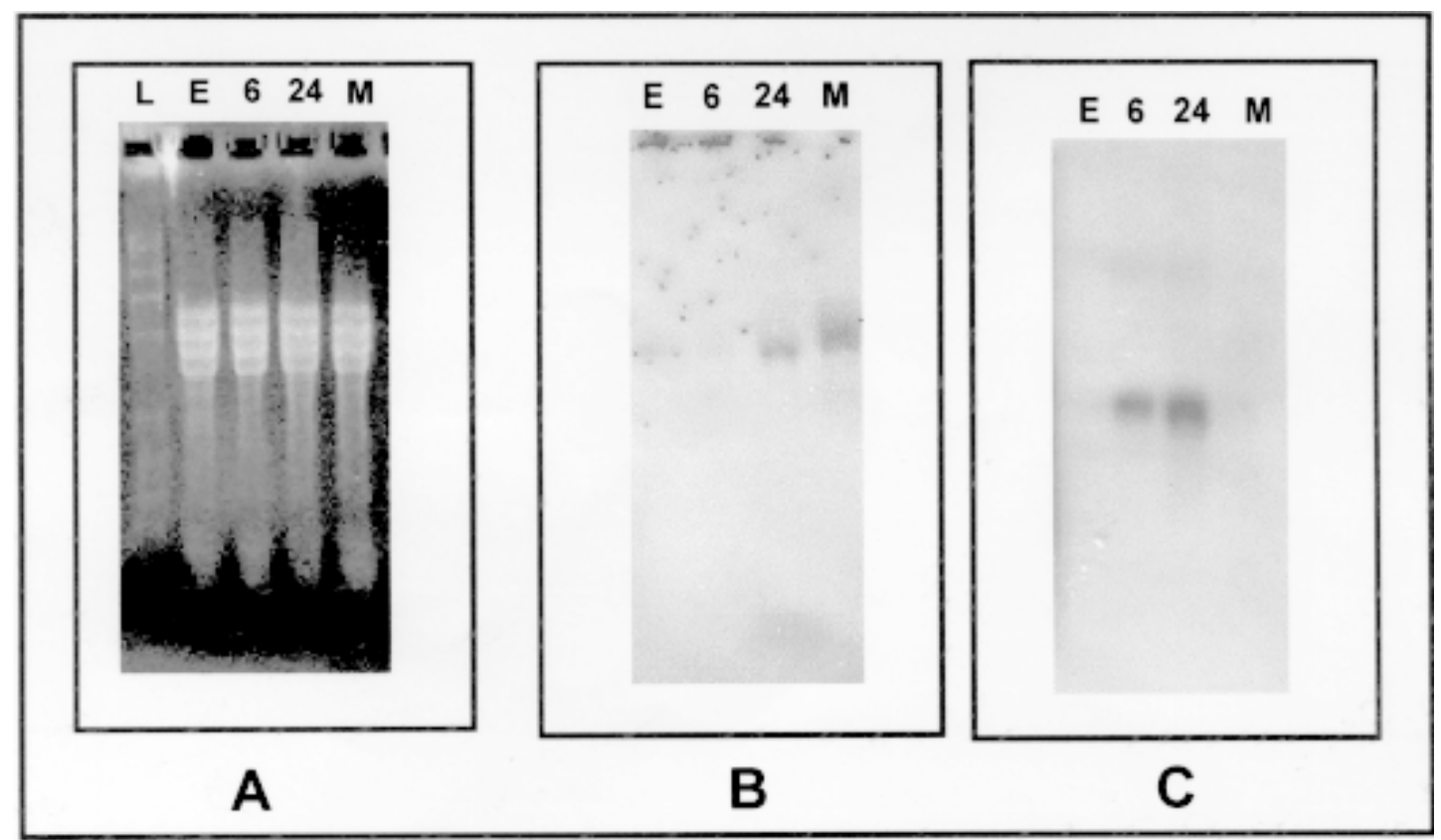

Fig. 2: Northern blot analysis of different stage specific clones selected during the metacyclogenesis process. Polysomal RNA from LIT medium epimastigotes (E), $6 \mathrm{hr}$ differentiating cells (6), $24 \mathrm{hr}$-differentiating cells (24) and metacyclic trypomastigotes (M) were electrophoresed on denaturing-agarose gels and blotted onto nylon membranes. The filters were accordingly treated and hybridized to the probes corresponding to the investigated clones. A: ethidium bromide stained agarose-gel; B: the RNA was transferred to a nylon membrane and probed with clone \#10, isolated using the representation of differential expression (RDE) procedure with metacyclic trypomastigotes polysomal RNA; C: the RNA was transferred to a nylon membrane and probed with clone \#24 isolated using the RDE procedure with polysomal RNA isolated from $6 \mathrm{hr}$ differentiating parasites. 
ferring to metacyclic trypomastigotes their stage specific properties. Consequently, the knockout of these genes might result in mutants incapable to differentiate or attenuated, that might be tested as live-vaccines against Chagas disease. On the other hand, it is possible that some of these stage specific genes encode enzymes that might proof to be new targets for the development of new drugs for Chagas disease chemotherapy.

\section{REFERENCES}

Bonaldo MC, Souto-Padron T, De Souza W, Goldenberg S 1988. Cell-substrate adhesion during Trypanosoma cruzi differentiation. J Cell Biol 106: 1349-1358.

Boothroyd JC, Cross GAM 1988 Transcripts coding for surface glycoproteins of Trypanosoma bruce $i$ have a short, identical exon at their 5' end. Gene 20: 218-289

Contreras VT, Morel CM, Goldenberg S 1985a. Stage specific gene expression precedes morphological changes during Trypanosoma cruzi metacyclogenesis. Molec Biochem Parasitol 14: 83-96.

Contreras VT, Salles J M, Thomas N, Morel CM, Goldenberg S 1985b. In vitro differentiation of Trypanosoma cruzi under chemically defined conditions. Molec Biochem Parasitol 16: 315-327.

De Souza W 1983. Cell Biology of Trypanosoma cruzi. Int Rev Citol 86: 197-283.

Furger A, Schürch N, Kurath U, Roditi I 1997. Elements in the 3' untranslated region of procyclin mRNA regulate expression in insect forms of Trypanosoma brucei by modulating RNA stability and translation. Mol Cell Biol 17: 4372-4380.

Krieger MA, Goldenberg S 1998. Representation of differential expression (RDE): A new approach to study differential gene expression in trypanosomatids. Parasitol Today 14: 163-166.

Lebowitz JH, Smith HQ, Rusche L, Beverley SM 1993. Coupling of poly(A) site selection and trans-splicing in Leishmania. Genes and Develop 7: 996-1007.

Liang P, Pardee AB 1992. Differential display of eukaryotic messenger RNA by means of the polymerase chain reaction. Science 257: 967-971.

Lisitsyn N, Lisitsyn N, Wigler M 1993. Cloning the differences between two complex genomes. Science 259: 946-951.

Muhich ML, Boothroyd JC 1988. Polycistronic transcripts in trypanosomes and their accumulation during heat shock: evidence for a precursor role in mRNA synthesis. Mol Cell Biol 8: 3837-3746.

Nozaki T, Cross GA 1995. Effects of 3' untranslated and intergenic regions on gene expression in Trypanosoma cruzi. Mol Biochem Parasitol 75: 55-67.

Ruef BJ, Dawson BD, Tewari D, Fouts DL, Manning JE 1994. Expression and evolution of members of the Trypanosoma cruzi trypomastigote surface antigen multigene family. Mol Biochem Parasitol 63: 109120.

Schenkman S, Eichinger D, Pereira ME, Nussenzweig V 1994. Structural and functional properties of Trypanosoma trans-sialidase. Аnnu Rev Microbiol 48: 499-523.

Teixeira SM, Russell DG, Kirchhoff LV, Donelson JE 1994. A differentially expressed gene family encoding "amastin," a surface protein of Trypanosoma cruzi amastigotes. J Biol Chem 269: 20509-20516.

Vanhamme L, Pays E 1995. Control of gene expression in trypanosomes. Microbiol Rev 59: 223-40. 